\title{
Dynamics-Aware Similarity of Moving Objects Trajectories
}

\author{
Goce Trajcevski $^{*} \quad$ Hui Ding $\quad$ Peter Scheuermann $^{\dagger}$ \\ Department of EECS Northwestern University \\ goce,hdi117,peters@eecs.northwestern.edu \\ Roberto Tamassia
Department of CS
Brown University
rt@cs.brown.edu \\ Dennis Vaccaro \\ Northrop Grumman Corp. \\ Defense Systems Division \\ dennis.vaccaro@ngc.com
}

\begin{abstract}
This work addresses the problem of obtaining the degree of similarity between trajectories of moving objects. Typically, a Moving Objects Database (MOD) contains sequences of (location,time) points describing the motion of individual objects, however, they also have an implicit information about the velocity, which is an important attribute describing the dynamics of a particular object. Our main goal is to extend the MOD functionalities with the capability of reasoning about how similar are the trajectories of objects that, possibly, move along geographically different routes. Towards this, we use a distance function which balances the lack of temporal-awareness of the Hausdorff distance with the generality (and complexity of calculation) of the Fréchet distance. Based on the observation that, as a firstapproximation in practice, the individual segments of trajectories are assumed to have constant speed, we provide efficient algorithms for: (1) optimal matching between trajectories; and (2) approximate matching between trajectories, both under translations and rotations, where the approximate algorithm guarantees a bounded error-quality with respect to the optimal one.
\end{abstract}

\section{INTRODUCTION AND MOTIVATION}

The management of the transient location-in-time information of mobile entities, is a fundamental ingredient for a variety of applications that involve Location Based Service (LBS) [25], and is a topic of the field known as Moving Ob-

\footnotetext{
* Research supported by the Northrop Grumman Corp., contract: P.O.8200082518

${ }^{\dagger}$ Research supported by the NSF grant IIS-0325144/003

$\ddagger_{\text {Research supported in part by NSF grant IIS-0324846 }}$
}

Permission to make digital or hard copies of all or part of this work for personal or classroom use is granted without fee provided that copies are not made or distributed for profit or commercial advantage and that copies bear this notice and the full citation on the first page. To copy otherwise, to republish, to post on servers or to redistribute to lists, requires prior specific permission and/or a fee.

ACM GIS 2007, Seattle, Washington USA

Copyright 200X ACM X-XXXXX-XX-X/XX/XX $\ldots \$ 5.00$. ject Databases (MOD) [19]. This work attempts to increase the MOD capabilities with providing algorithmic solutions that can be used to answer queries such as:

- Q1: "Retrieve all the troops which had similar motion patterns in the geographic regions $R 1$ and $R 2$ in the last two months."

- Q2: Retrieve all the trajectories that had similar motion patterns while looking for parking in the weekends around the shopping malls in the Mid-West."

- Q3: "Retrieve all the Hurricanes with similar trajectories that occurred in any US state along the Mexican Gulf in the last 5 years."

The commonality of all these queries is that the motion of interest need not occur in the same geo-locations (e.g., the hurricanes may occur in Texas and Florida) and, even more importantly, need not follow similar routes in terms of the geo-coordinates (e.g., a hurricane moving North and then changing its direction to North-West may be very similar to the one that has moved East and changed its direction to North-East).

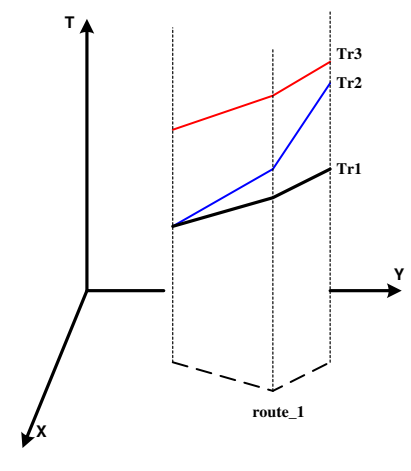

a.) Dynamics over same route

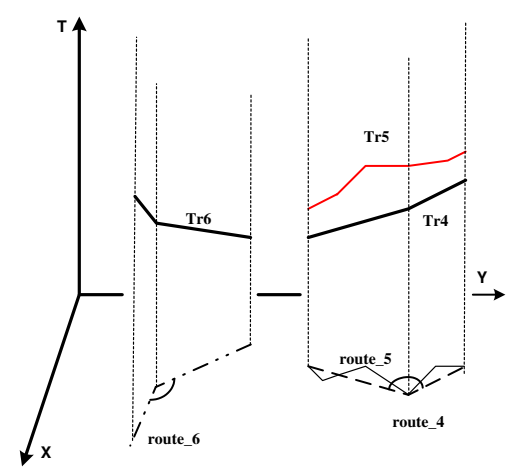

b.) Dynamics over different routes
Figure 1: Similarities of Motions

The main idea behind the similarity of trajectories that we tackle in this work is the ability to compare the relative motion of the moving objects along the consecutive segments of their corresponding trajectories. An illustration of our desiderata is provided in Figure 1. Namely, Figure 1.a) il- 
lustrates three trajectories $T r_{1}, T r_{2}$ and $T r_{3}$, corresponding to the motion plans of three objects, say, $o_{1}, o_{2}$ and $o_{3}$, all of which were moving along a same $2 \mathrm{D}$ route (illustrated as route_1). However, although, $o_{1}$ and $o_{2}$ begin their trips at a same time, their respective trajectories are very different - namely, $o_{2}$ has been moving much slower than $o_{1}$. Throughout this work, we would like to perceive such trajectories as not similar. On the other hand, although $o_{3}$ had started its trip later than $o_{1}$ (and $o_{2}$ ), its trajectory, $\operatorname{Tr}_{3}$ exhibits the same dynamics (velocity patterns over the corresponding route segments) as $T r_{1}$. Clearly, the only difference is that $T r_{3}$ is translated in the temporal domain with respect to $T r_{1}$ and we would like to consider them similar. Figure 1.b) illustrates some other discrepancies among trajectories that we would like to consider acceptable for the purpose of similarity. Namely, although the route of $\operatorname{Tr}_{5}$ does not exactly match the route of $T r_{4}-$ e.g., several small detours were taken by the respective object $O_{5}$, the duration of both trips is approximately equal and the spatial deviations are not too large (plus we do need a translation in the temporal dimension). A careful observation of $\operatorname{Tr}_{6}$ reveals that although it has a completely different $2 \mathrm{D}$ route from $T r_{1}$, its corresponding segments are of equal length and are having equal corresponding angles between themselves. Moreover, the speed of the moving object, say $o_{6}$, along the route-segments of $\mathrm{Tr}_{6}$ is exactly the same as the one of the corresponding object, say $o_{4}$, along the segments of $\operatorname{Tr}_{4}$.

One of the main contributions of this work is precisely the introduction of the concept of dynamical similarity of trajectories under the rigid motion transformations, that is, translations and rotations [5, 17]. Translation is allowed both in the spatial and temporal dimension, while rotation is allowed only in the $2 \mathrm{D}$ - spatial dimension. We use an intuitive similarity-distance function that enables us to capture the relative dynamics of the trajectories, which can be qualified as a special case of the Fréchet distance $[5,6]$ for polygonal curves, and does not suffer from the timeunawareness of the Hausdorff distance [5, 11]. We present efficient algorithmic solutions to two aspects of the problem of similarity between trajectories: (1.) optimization: finding the rigid transformation (rotation/translation in the spatial domain, and translation in the temporal domain) which minimizes the similarity-distance between two given trajectories, and (2.) approximation: determining an approximate matching between two trajectories using a fasterthan-optimal algorithm, with a guarantee that its output is within a fixed error-bound from the optimal similaritydistance. Additionally, our algorithms can also be used for determining whether one of the trajectories is similar to a portion of the other.

The rest of this paper is structured as follows. In Section 2 we recollect the necessary background. Section 3 presents our main results - the algorithms for determining the optimal and approximate similarity matching between trajectories. In Section 4 we present experimental observations which quantify the benefits of our proposed methods. Section 5 positions our work with respect to the related literature and Section 6 concludes the paper and presents directions for future work.

\section{PRELIMINARY BACKGROUND}

We now define the concepts that will be used in the rest of this paper. Firstly, we present a definition of a trajectory, which is often used in the MOD literature $[19,27,28]$ to describe the motion of the moving objects, assuming that the objects are moving in a $2 \mathrm{D}$ space with respect to a given coordinate system:

Definition 1. A trajectory $\operatorname{Tr}$ is a function $F_{t}: T \rightarrow \mathcal{R}^{2}$ which maps a given (temporal) interval $\left[t_{b}, t_{e}\right]$ into a onedimensional subset of $\mathcal{R}^{2}$. It is represented as a sequence of $3 D$ points (2D geography + time) $\left(x_{1}, y_{1}, t_{1}\right),\left(x_{2}, y_{2}, t_{2}\right)$, $\ldots,\left(x_{n}, y_{n}, t_{n}\right)$, where $t_{b}=t_{1}$ and $t_{e}=t_{n}$ and $t_{1} \leq t_{2} \leq$ $\ldots \leq t_{n}$

Each point $\left(x_{i}, y_{i}, t_{i}\right)$ in the sequence represents the $2 \mathrm{D}$ location $\left(x_{i}, y_{i}\right)$ of the object, at the time $t_{i}$. For every $t \in\left(t_{i}, t_{i+1}\right)$, the location of the object is obtained by a linear interpolation between $\left(x_{i}, y_{i}\right)$ and $\left(x_{i+1}, y_{i+1}\right)$ with the ratio $\left(t-t_{i}\right) /\left(t_{i+1}-t_{i}\right)$, which is, in between two points the object is assumed to move along a straight line-segment and with a constant speed. The $2 \mathrm{D}$ projection of $\mathrm{Tr}$ is a polygonal chain with vertices $\left(x_{1}, y_{1}\right),\left(x_{2}, y_{2}\right) \ldots\left(x_{n}, y_{n}\right)$ and it is called a route of Tr.

Observe that a trajectory may represent both the past and the future motion, i.e. the motion plan of a given object (c.f. [19]). Typically, for future trajectories, the user provides the starting location, starting time and the destination (plus, possibly, a set of to-be-visited) points, and the MOD server uses these, along with the distribution of the speed-patterns on the road segments as inputs to a dynamic extension of the Dijkstra's algorithm [13, 27], to generate the shortest traveltime trajectory. As an example of the usage of this model, aside from the commercial-fleet vehicles (e.g., FedEx), the monthly requests for shortest travel-time individual futuretrajectories posed to MapQuest is 46.4 million, Yahoo!Maps - 20 million, and Google Maps - 19.1 million [23].

We also need to recollect some definitions from the Computational Geometry (CG) literature $[5,6]$ regarding the distance(s) between curves. In what follows, we consider $2 \mathrm{D}$ planar curves, given as continuous mappings $C_{i}:\left[a_{i}, b_{i}\right] \rightarrow$ $\mathcal{R}^{2}$ (i.e., via parametrization), where $a_{i}<b_{i}(\in \mathcal{R})$. Naturally, for our purposes, the domain of $C_{i}$ will correspond to the temporal dimension.

Definition 2. Let $C_{1}:\left[a_{1}, b_{1}\right] \rightarrow \mathcal{R}^{2}$ and $C_{2}:\left[a_{2}, b_{2}\right] \rightarrow$ $\mathcal{R}^{2}$ denote two curves. Let $\|\cdot\|$ denote the $L_{2}$ norm and let $P_{1}$ (resp. $\left.P_{2}\right)$ denote a point on $C_{1}$ (resp. $C_{2}$ ). The Hausdorff distance from between $C_{1}$ and $C_{2}$ is defined as:

$$
\begin{aligned}
& \delta_{H}\left(C_{1}, C_{2}\right):=\max \left(\tilde{\delta}_{H}\left(C_{1}, C_{2}\right), \tilde{\delta}_{H}\left(C_{2}, C_{1}\right)\right), \text { where } \\
& \tilde{\delta}_{H}\left(C_{1}, C_{2}\right)=\sup _{P_{1} \in C_{1}} \inf _{P_{2} \in C_{2}}\left\|P_{1}-P_{2}\right\| \\
& \text { (resp. } \left.\tilde{\delta}_{H}\left(C_{2}, C_{1}\right)=\sup _{P_{2} \in C_{2}} \inf _{P_{1} \in C_{1}}\left\|P_{1}-P_{2}\right\|\right)
\end{aligned}
$$

and $\tilde{\delta}_{H}\left(C_{1}, C_{2}\right)$ (resp. $\tilde{\delta}_{H}\left(C_{2}, C_{1}\right)$ ) denotes the directed Hausdorff distance from $C_{1}$ to $C_{2}$ (resp. from $C_{2}$ to $C_{1}$ ).

The Hausdorff distance between two curves simply looks for the smallest $\varepsilon$ such that $C_{1}$ is completely contained in the $\varepsilon$-neighborhood of $C_{2}$ (i.e., $C_{1}$ is completely contained in the Minkowski Sum [12] of $C_{2}$ and a disk with radius $\varepsilon$ ) and vice versa. Although it is arguably a very natural distance measure between curves and/or compact sets, the Hausdorff distance is too "static", in the sense that it neither considers any direction nor any dynamics of the motion along the curves. A classical example of the inadequacy of the Hausdorff distance, often used in the CG literature $[5,6,17]$ is the "man walking the dog". Figure 2 illustrates the corresponding routes of the man (M-route) and the $\operatorname{dog}$ (D-route), as 


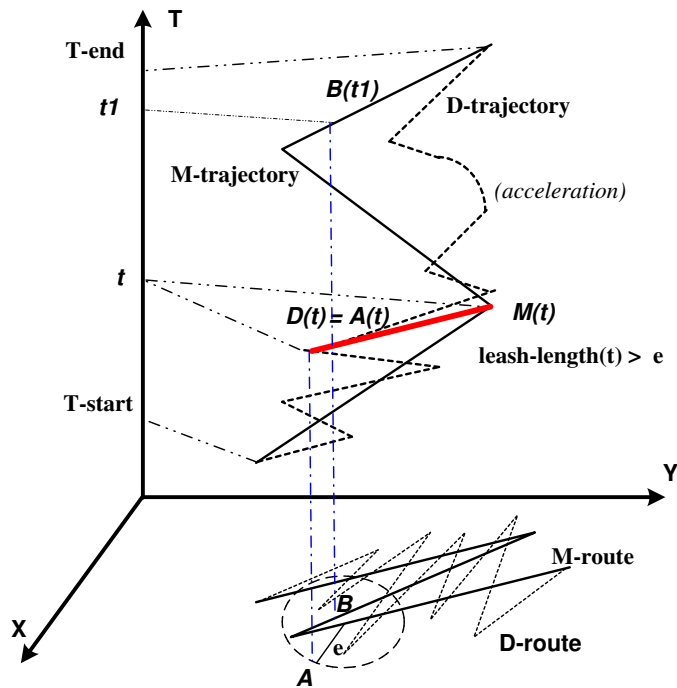

Figure 2: Hausdorff vs. Fréchet distance

well as their trajectories $M$-trajectory and $D$-trajectory. Observe that, ignoring the temporal aspect of their motions, the D-route and the M-route are within Hausdorff distance of $e$, as exemplified by the points $A$ and $B$ in the $X-Y$ plane. However, their actual (temporally-aware) distance corresponds to the minimal length of the leash that the man needs to hold. The 3D part of Figure 2 illustrates the discrepancy between the distances among the points along the routes, and their corresponding counterparts along trajectories: when the $\operatorname{dog}$ is at the point $A$, which is at time $t$, the man is actually at $M(t)$, and their distance is much greater then $e$ (the man is at the geo-location $B$ at the time $t_{1}>t$ ). Now we have the following:

Definition 3. Let $C_{1}:\left[a_{1}, b_{1}\right] \rightarrow \mathcal{R}^{2}$ and $C_{2}:\left[a_{2}, b_{2}\right] \rightarrow$ $\mathcal{R}^{2}$ denote two curves, and let $\|\cdot\|$ denote the $L_{2}$ norm. The Fréchet distance of $C_{1}$ and $C_{2}$, denoted by $\delta_{F}\left(C_{1}, C_{2}\right)$ is defined as: $\quad \delta_{F}\left(C_{1}, C_{2}\right):=\inf _{\alpha, \beta} \max _{t \in[0,1]}\left\|C_{1}(\alpha(t))-C_{2}(\beta(t))\right\|$ where $\alpha$ (resp. $\beta$ ) ranges over all the possible continuous and monotonically increasing functions $[0,1] \rightarrow\left[a_{1}, b_{1}\right]$ (resp. $\left.[0,1] \rightarrow\left[a_{2}, b_{2}\right]\right)$.

The Fréchet distance is more general than the Hausdorff one, in the sense that it allows for a variety of possible motion-patterns along the given route-segments, as formalized by the functions $\alpha$ and $\beta$ in Definition 3. As an illustration, observe that on the portion of the D-trajectory, the dog may be moving non-uniformly (i.e., accelerating) along a route segment. Recall, however, that this is precluded in the Definition 1 for trajectories in MOD settings. It can be verified (c.f. [2]) that for any two curves $C_{1}$ and $C_{2}$ it holds that $\delta_{H}\left(C_{1}, C_{2}\right) \leq \delta_{F}\left(C_{1}, C_{2}\right)$. Observe that in Definition 3, the requirement is that both $\alpha$ and $\beta$ are increasing functions. If this is relaxed, then the distance function is called weak Fréchet distance, denoted by $\tilde{\delta}_{F}\left(C_{1}, C_{2}\right)$, which intuitively corresponds to the ability of both the man and the dog moving backwards along the routes and: $\delta_{H}\left(C_{1}, C_{2}\right) \leq \tilde{\delta}_{F}\left(C_{1}, C_{2}\right) \leq \delta_{F}\left(C_{1}, C_{2}\right)$ [2].

\section{DYNAMICS-AWARE SIMILARITY}

Our main goal is to obtain and efficiently calculate a distance-measure which captures a relativized space+time difference of two given trajectories, for the purpose of measuring the similarity of their corresponding motions. Firstly, we present the necessary setup and introduce the distance function used in this work. Subsequently, we focus on the case where the trajectories have equal temporal durations and we analyze in details the optimization of matching two trajectories under translations and rotations in the spatial domain. We address separately the problem of optimal and approximate matchings. Lastly, we demonstrate how our techniques can be used for comparing the similaritybased containment of trajectories with different temporaldurations. Due to space limitations, we only give brief justification of our claims instead of formal proofs.

\subsection{Similarity Distance of Trajectories}

Let $\operatorname{Tr}_{1}=\left[\left(x_{11}, y_{11}, t_{11}\right),\left(x_{12}, y_{12}, t_{12}\right), \ldots,\left(x_{1 n}, y_{1 n}, t_{1 n}\right)\right]$ and $\operatorname{Tr}_{2}=\left[\left(x_{21}, y_{21}, t_{21}\right),\left(x_{22}, y_{22}, t_{22}\right), \ldots\left(x_{2 m}, y_{2 m}, t_{2 m}\right)\right]$ denote two trajectories with $n$ and $m$ segments, respectively. When measuring the distance between the two objects whose motions are represented by the $T r_{1}$ and $T r_{2}$, we would like to know its values at given time-points. The intuitive reason is that one cannot say "the distance between $o_{1}$ and $o_{2}$ is $d$, when $o_{1}$ is at location $L_{1}\left(x_{1}, y_{1}\right)$ and $o_{2}$ is at location $L_{2}\left(x_{2}, y_{2}\right)$, except, $o_{1}$ was at $L_{1}$ at time $t_{1}, o_{2}$ was at $L_{2}$ at time $t_{2}$, and $t_{1}$ and $t_{2}$ are far apart". This observation was actually used as a basis for introducing the, so called, Eucledean time-Uniform distance function in [11], a variant of which is given by the following:

Definition 4. Given two trajectories $\operatorname{Tr}_{1}$ and $T r_{2}$, the Eucledean time-Uniform distance function

$E_{u d}:\left(t, T r_{1}, T r_{2}\right) \rightarrow \mathcal{R}$ is defined as follows:

$E_{u d}\left(t, T r_{1}, T r_{2}\right)=\|P(t)-Q(t)\| \quad$ when $t \in\left(\left[t_{11}, t_{1 n}\right] \cap\right.$ $\left.\left[t_{21}, t_{2 m}\right]\right)$ and it is undefined otherwise. $P(t)$ (resp. $\left.Q(t)\right)$ is the $2 D$ point corresponding to the spatial locations on the trajectory $\operatorname{Tr}_{1}$ (resp. $T r_{2}$ ) at time $t$.

The Eucledean time-Uniform Distance of two trajectories $\operatorname{Tr}_{1}$ and $T r_{2}$ is defined as

$$
T E_{u d}\left(T r_{1}, T r_{2}\right)=\max _{t} E_{u d}\left(t, T r_{1}, T r_{2}\right)
$$

In other words, the $T E_{u d}$ distance between trajectories maximizes the value of $\sqrt{(P . x-Q \cdot x)^{2}+(P . y-Q \cdot y)^{2}}$, taken at same time-instances over their common temporal interval.

When two trajectories correspond to trips of equal temporal duration, $\left(t_{1 n}-t_{11}=t_{2 m}-t_{21}\right)$, we can always "transform" one of them by translating it in the temporal domain. Hence, without loss of generality, we will assume in what follows that the two trajectories $T r_{1}$ and $T r_{2}$ pertain to same temporal intervals, i.e., $t_{11}=t_{21}$ and $t_{1 n}=t_{2 n}$. For the purpose of calculating $T E_{u d}\left(T r_{1}, T r_{2}\right)$ we rely on the following:

Lemma 1. Given two trajectory segments over the same temporal interval: $\left[P_{1} P_{2}\right]=\left[\left(x_{11}, y_{11}, t_{1}\right),\left(x_{12}, y_{12}, t_{2}\right)\right]$ and $\left[Q_{1} Q_{2}\right]=\left[\left(x_{21}, y_{21}, t_{1}\right),\left(x_{22}, y_{22}, t_{2}\right)\right]$, the maximal value of $E_{u d}\left(t, T r_{1}, T r_{2}\right)$ is obtained at one of the corresponding endpoints, i.e., either $T E_{u d}\left(T r_{1}, T r_{2}\right)=\left\|\overline{P_{1} Q_{1}}\right\|$ or $T E_{u d}\left(T r_{1}, T R_{2}\right)=\left\|\overline{P_{2} Q_{2}}\right\|$.

The proof is based on the analysis of $\frac{d E_{u d}(t)}{d t}$ and $\frac{d^{2} E_{u d}(t)}{d t^{2}}$.

Recalling Definition 3 (Section 2), one may observe that the $T E_{u d}$ distance function is actually a special case of the Fréchet distance when $\alpha$ and $\beta$ are restricted to the natural 
(linear) mapping $[0,1] \rightarrow\left[t_{11}, t_{1 n}\right]$. Now, one of the main questions of this work can be formulated as follows: when two trajectories $T r_{1}$ and $T r_{2}$ are ranging over the same temporal interval, what is the minimal value of $T E_{u d}\left(T r_{1}, T r_{2}^{\prime}\right)$, where $T R_{2}^{\prime}$ is obtained by applying only translation and rotation to $T r_{2}$ ? Formally:

DeFinition 5. Given two trajectories $T r_{1}$ and $T r_{2}$, their Rigid Transformations Similarity Distance $R T S D\left(T r_{1}, \operatorname{Tr}_{2}\right)$ is defined as $R T S D\left(T r_{1}, \operatorname{Tr}_{2}\right)=\left(T E_{u d}\left(T r_{1}, \Psi \circ \Phi\left(T r_{2}\right)\right)\right)$, where $\Psi \circ \Phi$ is a composition of translations $(\Psi)$ and rotations $(\Phi)$ in a horizontal plane, applied to the route of $\mathrm{Tr}_{2}$.

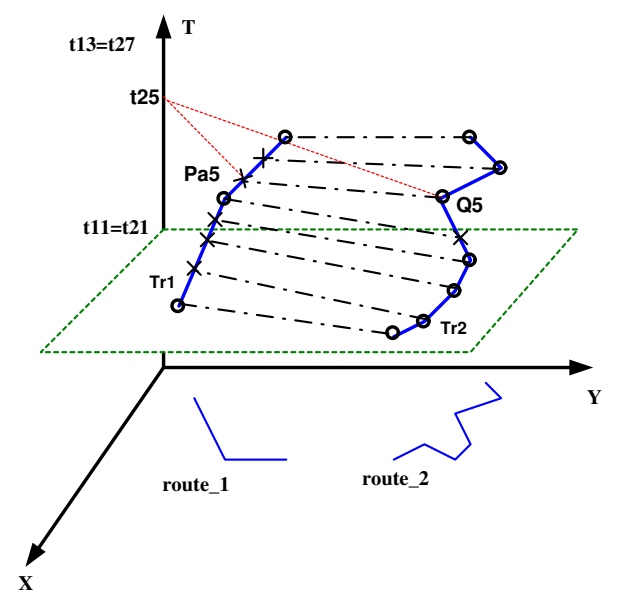

Figure 3: Artificial Vertices of Trajectories

\subsubsection{Optimal Similarity Matching}

In order to calculate the optimal $\operatorname{RTSD}\left(T r_{1}, T r_{2}\right)$ we need to determine the particular $\Psi_{\text {opt }}$ and $\Phi_{\text {opt }}$ that minimize the $T E_{u d}\left(T r_{1}, \Psi_{\text {opt }} \circ \Phi_{\text {opt }}\left(T r_{2}\right)\right)$, for which we proceed as follows. First, in each of $T r_{1}$ and $T r_{2}$ we introduce extra artificial vertices, so that they both have the same number of points. Essentially, if a given vertex of $\operatorname{Tr}_{1}$, say $\left(x_{1 k_{i}}, y_{1 k_{i}}, t_{1 k_{i}}\right)$ does not have a matching vertex on $\mathrm{Tr}_{2}$ at the same time-instance $t_{1 k_{i}}$, we introduce an artificial one $\left(x_{2 k_{i}}^{a}, y_{2, k_{i}}^{a}, t_{1 k_{i}}\right)$, and similarly for the vertices of $\mathrm{Tr}_{2}$. This is illustrated in Figure 3 where, originally, $T r_{1}$ has three vertices and $T r_{2}$ has seven, indicated with "०", however, after introducing the artificial ones, indicated with " $x$ ", each of them has eight vertices. For instance, $Q_{5}$ at $t_{25}$ is a genuine vertex of $\operatorname{Tr}_{2}$ and its corresponding artificial vertex on $T r_{1}$ is $P_{a 5}$ at that time. According to Definition 1, the spatial coordinates of each artificial vertex $\left(x_{k_{i}}^{a}, y_{k_{i}}^{a}\right)$ are obtained by a linear interpolation between the two original consecutive vertices $\left(x_{i}, y_{i}, t_{i}\right)$ and $\left(x_{(i+1)}, y_{(i+1)}, t_{(i+1)}\right)$, for which $t_{k_{i}} \in\left(t_{i}, t_{(i+1)}\right)$. Hence, we obtain two augmented trajectories $\operatorname{Tr}_{1}^{a}$ and $T r_{2}^{a}$ with exactly the same number of points $(O(m+n))$, pairwise matching in the temporal dimension. Now, as a generalization of Lemma 1, we know that $R T S D\left(T r_{1}, T r_{2}\right)$ will be obtained at one of the temporally-corresponding pairs of vertices in $T r_{1}^{a}$ and $T r_{2}^{a}$. As a consequence, the problem of calculating $R T S D\left(T r_{1}, T r_{2}\right)$ is now reduced to calculating the corresponding $L_{2}$ distances between two point-sets corresponding to the (2D projections of the augmented trajectories) vertices along the routes. Moreover, if we view the two sets of 2 D points $P=\left\{p_{1}, p_{2}, \ldots p_{u}\right\}$ and $Q=\left\{q_{1}, q_{2}, \ldots, q_{u}\right\}$, we have the restriction that the mapping $g: P \rightarrow Q$ is fixed, in the sense that $g\left(p_{j}\right)=q_{j}(j \in\{1,2, \ldots u\})$ where $u=O(m+n)$. Now we are interested in a composition $\Psi \circ \Phi$ such that the largest distance between $p_{j}$ and $\Psi \circ \Phi\left(q_{j}\right)$ is minimized over all $j$.

However, this particular problem, the optimal matching of two point-sets with a fixed mapping between them, was addressed in [22]. To explain the solution, observe firstly that applying a rotation $\rho$ around the coordinate-center with an angle $\theta$ to the point $q_{j}\left(x_{q j}, y_{q j}\right)$ will transform it to: $\rho_{\theta}\left(q_{j}\right)=\left(x_{q j} \cos \theta-y_{q j} \sin \theta, x_{q j} \sin \theta+y_{q j} \cos \theta\right)$. Subsequently, translating it by a vector $\vec{\tau}=\left(x_{\tau}, y_{\tau}\right)$, brings it to the point $\tau\left(\rho_{\theta}\left(q_{j}\right)\right)=\left(\left(x_{q j} \cos \theta-y_{q j} \sin \theta\right)+x_{\tau},\left(x_{q j} \sin \theta+\right.\right.$ $\left.\left.y_{q j} \cos \theta\right)+y_{\tau}\right)$ (c.f. [22]). Hence, our original problem of obtaining the $R T S D\left(T r_{1}, T r_{2}\right)$ is reduced to finding $\theta$ and $\vec{\tau}$ such that $\max \| p_{j}-\tau\left(\rho_{\theta}\left(q_{j}\right) \|\right)$ is minimized. Equivalently, we are seeking: $\min _{\theta, \tau} \max _{j} f_{j}\left(\theta, x_{\tau}, y_{\tau}\right)=\min _{\theta, \tau} \max _{j}$

$\left[\left(x_{p j}-\left(\left(x_{q j} \cos \theta-y_{q j} \sin \theta\right)+x_{\tau}\right)\right)^{2}+\left(y_{p j}-\left(\left(x_{q j} \sin \theta+\right.\right.\right.\right.$ $\left.\left.\left.\left.y_{q j} \cos \theta\right)+y_{\tau}\right)\right)^{2}\right]$.

For a fixed value of $\theta$, say $\theta^{\prime}$, we are essentially looking at $\min _{x_{\tau}, y_{\tau}} \max _{j} f_{j}\left(\theta^{\prime}, x_{\tau}, y_{\tau}\right)$, and the problem is now reduced to the one of minimum enclosing circle [24]. As $\theta$ "moves" between 0 and $2 \pi$, the problem of calculating the $R T S D\left(T r_{1}, T r_{2}\right)$ becomes the one of finding the minimum enclosing circle for "moving points" $\rho_{\theta}\left(q_{j}\right)$, subject to having a pair of points $\rho_{\theta}\left(q_{s}\right)$ and $\rho_{\theta}\left(q_{l}\right)$ on its boundary. The problem of the minimum enclosing circle can be reduced to finding the minimum value on the envelope of a family of functions $f_{j}\left(\theta^{\prime}, x_{\tau}, y_{\tau}\right)$. Its relationship with the $d y$ namic furthest Voronoi diagram [8] of the points $\left(x_{q j} \cos \theta-\right.$ $\left.y_{q j} \sin \theta\right)+x_{\tau},\left(x_{q j} \sin \theta+y_{q j} \cos \theta\right)+y_{\tau}$ is presented in details in [22]. Essentially, the algorithm fixes a pair of indices $s$ and $l$ and solves the constrained problem of finding the $\theta$ which yields the minimum enclosing circle having $\rho_{\theta}\left(q_{j}\right)$, subject to having a pair of points $\rho_{\theta}\left(q_{s}\right)$ and $\rho_{\theta}\left(q_{l}\right)$ on its boundary. For a fixed pair $l$ and $s$ (c.f. Lemma 4.1 in [22]) the complexity of obtaining the maximum diagram of $u$ functions of the kind $f_{j}\left(\theta^{\prime}, x_{\tau}, y_{\tau}\right)(1 \leq j \leq u, s \neq j \neq l)$ is $O\left(\lambda_{7}(u) \log u\right)$. Repeating the search for the minimum enclosing circle for all the possible pairs $s$ and $l$ on its boundary (and picking the smallest one among them) yields a complexity of $O\left(u^{2} \lambda_{7}(u) \log u\right)$. In the preceding, the $\lambda_{7}(u)$ denotes the maximum length of the $(u, 7)$ Davenport-Schinzel (DS) sequence, where the length of any $(u, a)$ DS sequence for $a>3(a \leq u)$ is $\lambda_{a}(u)=u \alpha(u)^{O\left(\alpha(u)^{a-3}\right)}$ (c.f. [26], where even sharper bounds are presented). $\alpha(u)$ denotes the extremely slow-growing inverse Ackermann function ${ }^{1}$. Hence, as a direct consequence of Theorem 4.1. in [22], the problem of calculating the optimal $R T S D\left(T r_{1}, T r_{2}\right)$ can be solved in $O\left((m+n)^{2} \lambda_{7}(m+n) \log (m+n)\right)$, where $\lambda_{7}(m+n)=(m+n) \alpha(m+n)^{O\left(\alpha(m+n)^{4}\right)}$.

\subsubsection{Approximate Similarity of Trajectories}

Often, instead of actually finding the optimal matching (minimizing RTSD) between two trajectories, one is interested in an approximate matching, which can be obtained by a faster algorithm. However, it is desirable to have a bound on the error of the obtained approximation, in

${ }^{1}$ We note that $\lambda_{1}(n)=n ; \lambda_{2}(n)=2 n-1$; and $\lambda_{3}(n)=$ $O(n \log n)[26]$. 
the following sense. Let $\varepsilon_{\text {opt }}=\operatorname{RTSD}\left(T r_{1}, T r_{2}\right)$, denote the distance obtained by finding the optimal transformation $\Psi_{\text {opt }} \circ \Phi_{\text {opt }}\left(\operatorname{Tr}_{2}\right)$. For a given approximate transformation $\Psi_{A} \circ \Phi_{A}\left(\operatorname{Tr}_{2}\right)$ - again, a composition of translation and rotation applied to $\mathrm{Tr}_{2}$, we say that it has a loss factor $d$ if $\varepsilon_{A}=E_{u d}\left(T r_{1}, \Psi_{A} \circ \Phi_{A}\left(T r_{2}\right)\right)$ satisfies the inequality $\varepsilon_{A} \leq d \cdot \varepsilon_{o p t}$ [17]. Equivalently, an approximation with error bound $\varepsilon_{A}$ is said to have a quality of " $c$ ", if it is within the bound of $(1+c) \varepsilon_{\text {opt }}[2,6]$.

Definition 6. Let $\operatorname{Tr}=\left[\left(x_{1}, y_{1}, t_{1}\right), \ldots,\left(x_{k}, y_{k}, t_{k}\right)\right] d e-$ note an arbitrary (actual or augmented) trajectory with $k$ points. The backbone of $\mathrm{Tr}$ is the line through the points $\left(x_{1}, y_{1}, t_{1}\right)$ and $\left(x_{k}, y_{k}, t_{k}\right)$. The backbone trajectory of $\mathrm{Tr}$, denoted BTr, is defined as follows:

- BTr begins at $\left(x_{1}, y_{1}, t_{1}\right)$ and ends at $\left(x_{k}, y_{k}, t_{k}\right)$.

- for each point $P_{i}=\left(x_{i}, y_{i}, t_{i}\right) \in T r, B T r$ has an anchorpoint $A P_{i}=\left(x_{i}^{a}, y_{i}^{a}, t_{i}^{a}\right)$, given by the intersection of the backbone with the horizontal plane through $P_{i}$, i.e., $t_{i}^{a}=t_{i}$ and $\left(x_{i}^{a}, y_{i}^{a}\right)$ is obtained by a linear interpolation between $\left(x_{1}, y_{1}\right)$ and $\left(x_{k}, y_{k}\right)$ at $t_{i}$. We call the segment $\overline{A P_{i} P_{i}}$ an anchor.

Let $T r_{1}=\left[P_{1}, \ldots, P_{k}\right]$ and $T r_{2}=\left[Q_{1}, \ldots, Q_{k}\right]$ denote two trajectories with the same number of points and spanning over identical time-intervals, i.e., $t_{P_{1}}=t_{Q_{1}}$ and $t_{P_{k}}=t_{Q_{k}}$. Let $B T r_{1}=\left[A P_{1}, A P_{2}, \ldots, A P_{k}\right]$ and $B T r_{2}=\left[A Q_{1}, \ldots, A Q_{k}\right]$ denote their respective backbone trajectories. We have:

Lemma 2. Let $\Psi$ and $\Phi$ denote a translation and rotation. If $T E_{u d}\left(T r_{1}, \Psi \circ \Phi\left(T r_{2}\right)\right) \leq \varepsilon$, then $T E_{u d}\left(B T r_{1}, \Psi \circ\right.$ $\left.\Phi\left(B T r_{2}\right)\right) \leq \varepsilon$.

Lemma 2 is a consequence of Definition 6 and the fact that the rotation and translation are rigid transformations, i.e., not only that they preserve the ratios, but also the lengths and angles. Since the first and the last anchor points coincide with the first and the last points on the actual trajectories, they are bound to be within distance $\varepsilon$ after $\Psi \circ \Phi$ is applied to $T r_{2}$, and the rest of the anchor points are linear combinations of the two end-points.

For a given backbone trajectory $B T r=\left[A P_{1}, \ldots, A P_{k}\right]$, we identify the direction from $A P_{1}$ towards $A P_{k}$ as a positive one which, in turn, defines the positive orientation along the $X-Y$ projection (route) of $B T r$, denoted by $B T r^{X-Y}$. With respect to the positive orientation, the $2 \mathrm{D}$ projection of a given anchor ${\overline{A P_{i} P_{i}}}^{X-Y}$ can be either on the left-hand side (LHS) or to the right-hand side (RHS) of the $B \operatorname{Tr}^{X-Y}$ and, for the special case when an anchor is collinear with $B \mathrm{Tr}^{X-Y}$, w.l.o.g we assume that it is on the RHS.

The concepts that we introduced so far are illustrated in Figure 4. Part a.) shows 2D projections of two trajectories which, after augmentation, i.e., introduction of artificial vertices, have 11 points each and, as one extreme case, all the points on each trajectory are on the RHS of their corresponding backbone-trajectories. Part b.), on the other hand, shows the 2D projections of (augmented) trajectories with 9 points each and, as another extreme case, they are completely on the opposite side of their backbone-trajectories. Before we proceed with the description of the algorithm for approximate matching between trajectories, we need the following:

Definition 7. Given two trajectories, $\operatorname{Tr}_{1}=\left[P_{1}, \ldots, P_{k}\right]$ and $\operatorname{Tr}_{2}=\left[Q_{1}, \ldots, Q_{k}\right]$, their $i$-th anchor discrepancy is defined as:

$\left\|\overline{A P_{i} P_{i}}-\overline{A Q_{i} Q_{i}}\right\|$, if $P_{i}^{X-Y}$ and $Q_{i}^{X-Y}$ are on the same side

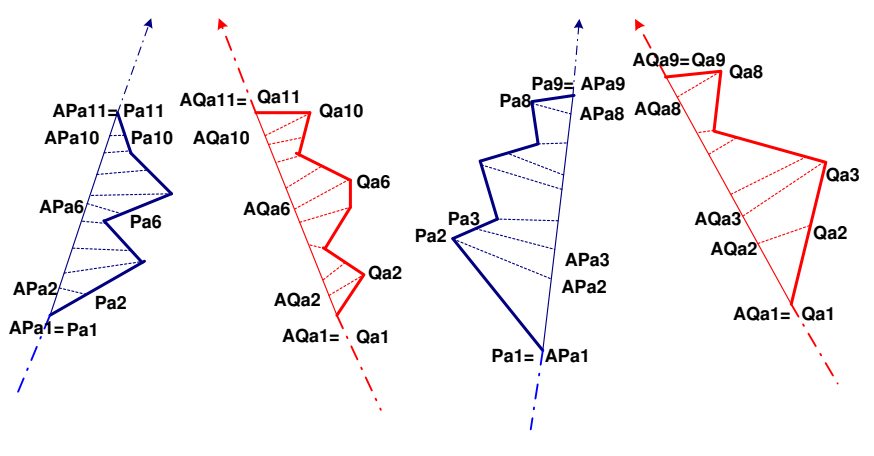

a.) Same orientation

b.) Opposite orientation

\section{Figure 4: Approximate Matching of Trajectories}

of the corresponding $B \operatorname{Tr}_{1}^{X-Y}$ and $B \operatorname{Tr}_{2}^{X-Y}$.

$\left\|\overline{A P_{i} P_{i}}+\overline{A Q_{i} Q_{i}}\right\|$, if $P_{i}$ and $Q_{i}$ are on the opposite sides of the corresponding BTr $r_{1}$ and $B T r_{2}$.

As an illustration, in Figure 4a.), the maximum anchor discrepancy is $\overline{A Q a_{6} Q a_{6}}-\overline{A P a_{6} P a_{6}}$. On the other hand, the maximum anchor discrepancy in Figure 4b.) is $\overline{A Q a_{3} Q a_{3}}+$ $\overline{\mathrm{APa}_{3} \mathrm{~Pa}_{3}}$. Actually, the goal of our heuristic (approximate matching algorithm) is to find a transformation, which is a composition of translation $\Psi_{a}$ that will contribute to minimizing the anchor discrepancy between the two trajectories; and a rotation $\Phi_{a}$ that will minimize the rotation-distance between the points on $T r_{1}$ and $\Psi_{a}\left(T r_{2}\right)$. Assuming that the input trajectories are already augmented so that they have equal number of points, and that the temporal translation has already been applied so that their time-intervals coincide, we have the following algorithm for approximate similarity matching between two trajectories:

\section{Algorithm MMAD}

Input: two trajectories $T r_{1}$ and $T r_{2}$

Output: translation $\Psi_{a}$ and rotation $\Phi_{a}$

1. For each pair of anchors $\overline{A P a_{i} P a_{i}}$ and $\overline{A Q a_{i} Q a_{i}}$

2. Let Translation(i): $\Psi_{i}=\left(\left(\left(A P a_{i} . x+P a_{i} . x\right) / 2-\left(A Q a_{i} . x+\right.\right.\right.$ $\left.\left.\left.Q a_{i} . x\right) / 2\right),\left(\left(A P a_{i} . y+P a_{i} . y\right) / 2-\left(A Q a_{i} . x+Q a_{i} . y\right) / 2\right)\right) / /$ trans-

late $\mathrm{Tr}_{2}^{a}$ so that the mid-points of the $i$-th anchors coincide

3. For each $\Psi_{i}$

3.1 Find the corresponding pair of points for which $\left\|P_{j}-\Psi_{i}\left(Q_{j}\right)\right\|$ is maximal

3.2 Let rotation angle: $\theta_{j}^{(i)}=$ the angle which makes the i-th anchor midpoints, $P_{j}$ and $\Psi_{i}\left(Q_{j}\right)$ collinear.

4. Return the pair $\left(\Psi_{i}, \Theta_{j}^{(i)}\right)$ for which $T E_{u d}\left(T r_{1}, \Psi_{i} \circ \Theta_{j}^{(i)}\left(T r_{2}\right)\right)$ is minimal

Clearly, the time-complexity of the Algorithm MMAD is $O(m+n)^{2}$, with an additional space requirement of $O(m+$ $n)$. Let $\varepsilon_{M M A D}=T E_{u d}\left(T r_{1}, \Psi_{A} \circ \Phi_{A}\left(T r_{2}\right)\right)$. We have the following:

LEMMA 3. Algorithm MMAD generates an approximate matching with the loss-factor of 4 , i.e., $\varepsilon_{M M A D} \leq 4 \varepsilon_{\text {opt }}$

As a consequence of Lemma 2, we have that the midpoints of each corresponding anchors are at the distance $\leq \varepsilon_{o p t}$ when $T r_{2}$ is transformed by $\Psi_{\text {opt }} \circ \Phi_{\text {opt }}$. Hence, making the midpoints of the anchors with the maximal discrepancy coincide in the Algorithm MMAD due to $\Psi_{M M A D}$ will perturb the rest of the points with respect to the optimal transformation by an extra $\leq \varepsilon_{o p t}$. Due to the rotation $\Phi_{M M A D}$ 
we have another loss of $\leq 2 \varepsilon_{\text {opt }}$. Considering that the extra "initial" distance $\leq \varepsilon_{o p t}$, we obtain the factor of 4 .

\subsection{Temporal Discrepancy of Trajectories}

In the temporal dimension, the main source of a discrepancy between two trajectories is the difference of the duration of the corresponding objects' trips: $\delta_{T D}\left(T r_{1}, T r_{2}\right)=$ $\left|\left(t_{1 n}-t_{11}\right)-\left(t_{2 m}-t_{21}\right)\right|$. Without loss of generality, assume that $\left(t_{1 n}-t_{11}\right) \geq\left(t_{2 m}-t_{21}\right)$ (i.e., $T r_{1}$ has a longer temporal duration than $\mathrm{Tr}_{2}$. Now, a variant of the problem of dynamics similarity of the objects' motions can be restated as: what is the portion of $\operatorname{Tr}_{1}$ that is most similar to $\operatorname{Tr}_{2}$ ?

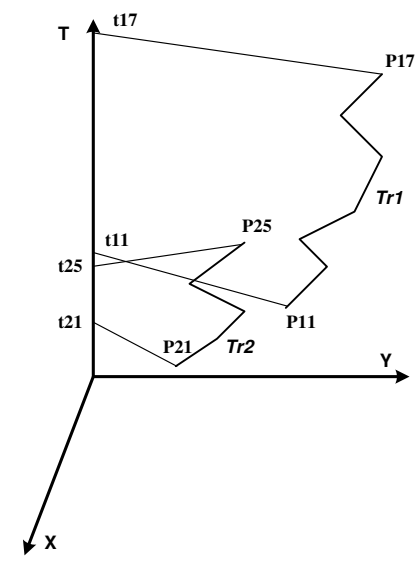

a.) Discrepancy between begin times (t11-t21) and end-times (t17-t25)

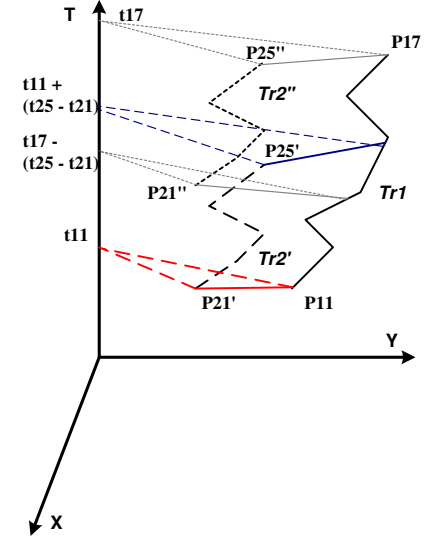

b.) "Temporal sliding" of trajectories
Figure 5: Temporal Distance Between Trajectories

Figure (5.a) illustrates the discrepancy between the begintimes and the end-times of two trajectories. Again, we observe that the "absolute" measures of the difference of the corresponding begin-points $\left(\left|t_{11}-t_{21}\right|\right)$ is not crucial for detecting their similarities, because a translation in the temporal domain can bring, say, $T r_{2}$ into $T r_{2}^{\prime}$ such that $t_{21}^{\prime}=t_{11}$. To be able to reason about the similarity between $T r_{2}$ and a portion of $T r_{1}$, we need to consider all the possible translations in the temporal domain. As an example of two extreme cases:

(1) The begin-times of two trajectories coincide - we shift the begin-point of $T r_{2}$ into $t_{11}$. Hence, $T r_{2}^{\prime}=\left[\left(x_{21}, y_{21},,\left(t_{21}+\right.\right.\right.$ $\left.\left.\left.\left(t_{11}-t_{21}\right)\right)\right), \ldots,\left(x_{2 m}, y_{2 m},\left(t_{2 m}+\left(t_{11}-t_{21}\right)\right)\right)\right]$;

(2) The end-times of two trajectories coincide - we shift the end-point of $\operatorname{Tr}_{2}$ into $t_{1 n}$. Hence, $\operatorname{Tr}_{2} "=\left[\left(x_{21}, y_{21,},\left(t_{21}+\right.\right.\right.$ $\left.\left.\left.\left(t_{1 n}-t_{2 m}\right)\right)\right), \ldots,\left(x_{2 m}, y_{2 m},\left(t_{2 m}+\left(t_{1 n}-t_{2 m}\right)\right)\right)\right]$. Figure (5.b) illustrates the concept of "possible translations" of $\mathrm{Tr}_{2}$ ("sliding") in the temporal dimension, and corresponding distances between points (e.g., $\overline{P_{21}^{\prime} P_{11}} ; \overline{P_{25} " P_{17}}$, etc.). Let $\tau_{t_{v}}\left(T r_{2}\right)$ denote a temporal translation of $T r_{2}$ for (onedimensional "vector") $t_{v}$ such that: $t_{21}+t_{v} \geq t_{11}$ and $t_{2 m}+t_{v} \leq t_{1 n}$. For each translation of $\tau_{t_{v}}$ in-between (and including) the two extreme cases, we are interested in the maximal $E_{u d}$ between a point on $\tau_{t_{v}}\left(T r_{2}\right)$ and a corresponding point on $T r_{1}$ which has a same time-value. Clearly, there are infinitely many values for $t_{v}$, however, as a consequence of Lemma 1, we only need to look at the discrete set of temporal translations $\tau_{t_{v}}$ which map a time-value of a vertex from $T r_{2}$ into a time-value of vertex of $T r_{1}$.

Definition 8. Let $T r_{1}$ and $T r_{2}$ denote two trajectories, where the temporal duration of $\mathrm{Tr}_{2}$ is $\leq$ than the temporal duration of $\operatorname{Tr}_{1}$. Their Temporal-Containment Similarity Distance TCSD is defined as

$$
\operatorname{TCSD}\left(T r_{1}, T r_{2}\right)=\min _{t v} \operatorname{RTS} D\left(T r_{1}, \tau_{t_{v}}\left(T r_{2}\right)\right)
$$

Based on the results in Section 3.1, and observing that due to Lemma 1 there is a total of $O(m n)$ temporal translations $\left(t_{v}\right)$ of interest, we have that:

(1.) The optimal $T C S D\left(T r_{1}, T r_{2}\right)$ can be obtained in $O\left(m n(m+n)^{2} \lambda_{7}(m+n) \log (m+n)\right)$;

(2.) An approximated $T C S D_{A}\left(T r_{1}, T r_{2}\right)$ with a loss-factor of 4 can be obtained in $O\left(m n(m+n)^{2}\right)$

\section{EXPERIMENTAL EVALUATION}

Our experiments were conducted on a PC with Pentium IV 3.0 GHz CPU, 1 Gigabytes of DDR2 memory and the Windows XP platform. We used both artificial and real data-sets. The artificial data set is generated using the network-based traffic generator [10] using the road network of Oldenburg. The trajectories in this data sets are temporally aligned and are of the same length. The realistic data set consists of 5000 trajectories of objects moving on the road segments of Cook County, Illinois. The length of these trajectories varies from 2 to 60 miles, and the average speed of the moving objects ranged between $10 \mathrm{mph}$ and $60 \mathrm{mph}$. Additionally, we generated a "perturbed" set of trajectories as follows. For each trajectory in the original dataset, we randomly selected a value of $\varepsilon$ and individual trajectory points were displaced by a randomly chosen value from the interval $[0.1 \varepsilon, \varepsilon]$, and in a random $X-Y$ direction.

Our first group of experiments focus on the quality of Algorithm MMAD. We compared the values $\varepsilon_{\text {approx }}$ obtained as an output of Algorithm MMAD with the values of $\varepsilon_{\text {opt }}$. More specifically, for each perturbed trajectory, we applied a randomly determined translation and rotation to obtain its variant of the perturbed trajectory. We then fed both the original trajectory and this variant into Algorithm MMAD to generate the approximate translation and rotation, and calculate the approximate distance. We measured the percentage of trajectories that yield an approximate distance within a given factor of $\varepsilon_{\text {opt }}$. The results are shown in the following table:

\begin{tabular}{cccccc}
\hline$\varepsilon_{\text {approx }} / \varepsilon_{\text {opt }}$ & 1.5 & 2.0 & 2.5 & 3.0 & 3.5 \\
\hline Trajectories & $92.7 \%$ & $98.5 \%$ & $99.2 \%$ & $99.68 \%$ & $100 \%$ \\
\hline
\end{tabular}

As can be seen, for more than $95 \%$ of the trajectories used in the experiments, Algorithm MMAD had a loss factor $\leq 2$, a loss factor $\leq 2.5$ in more than $99 \%$ cases.

Our next group of experiments studied the selectivity of the $T E_{u d}$ distance function for trajectories that have the same or similar routes. We picked a random trajectory $T r_{p i c k}$ from the Cook County data set and retrieved all the trajectories from the data set that have routes within a spatial (2-D Euclidian) distance of $\varepsilon_{S}$. For a filtering speed-up, the trajectories are indexed using an R-tree and we used the Linear Referencing System (LRS) of Oracle 9i [25] to measure the $T E_{u d}$ distance during the refinement stage. Subsequently, from that subset, we obtained the trajectories whose dynamics are similar to $T r_{p i c k}$ and are within RTSD value of $\leq \varepsilon_{S}$, applying the proper translation/shifting in the temporal domain. For trajectories whose temporal duration are not the same, we measured the TCSD best-fit among all the possible translations of the shorter-duration trajectory into 
the one with a longer duration. In the experiments, we varied the value of $\varepsilon_{S}$ from 0.5 to 2.5 miles and we averaged the results over 200 different choices of $T r_{\text {pick }}$.

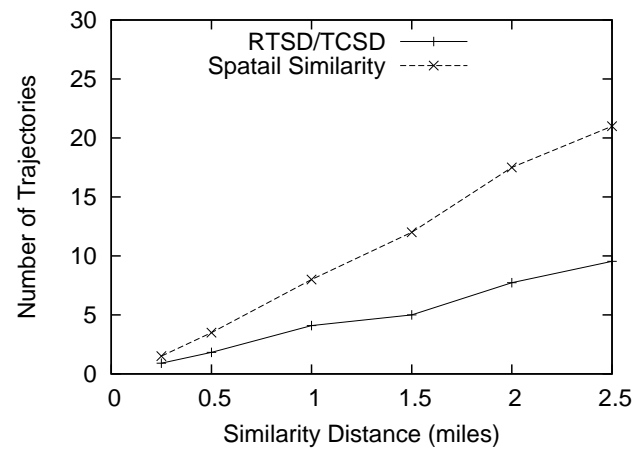

Figure 6: Trajectory Similarity vs. Route Similarity

As illustrated in Figure 6, the number of false-negatives can be quite large when the speed of the motion is not properly considered in the temporal dimension, and it increases in a linear manner with the value of $\varepsilon_{S}$. This illustrates the importance of our distance measure when considering the spatio-temporal similarities among moving object trajectories, as opposed to the pure spatial proximity of the moving objects' routes $[9,14]$.

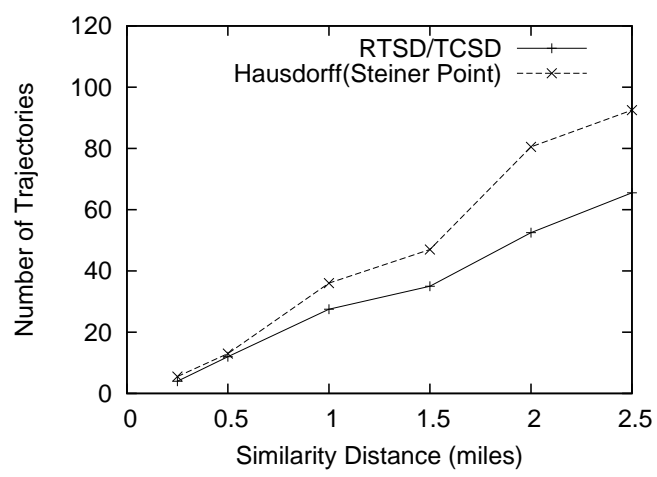

Figure 7: Dynamics-Aware vs. Steiner-point

Our last group of experiments measured the quality of our proposed techniques when, additionally, rigid transformations (translations + rotations) were applied in the spatial domain. A popular approach for matching 2-D polygonal shapes with respect to the Hausdorff distance is the one based on a reference point [3], relying on the Steiner point of compact 2-D sets. The work of [6] demonstrated that any point on a polygonal chain has a loss factor of 2 for the general Fréchet distance under translations only, however, there is no analogue of it for rotations. In the case of the 2-D routes of trajectories, we used the Steiner point calculated for the convex hull of the $2 \mathrm{D}$ projections of the points from the original trajectory and the anchor points of their corresponding backbones. As for rotation, we aligned every possible pair of respective points, and used the angle that would minimize the Hausdorff distance between the corresponding routes. Again, we randomly chose a trajectory $T r_{p i c k}$, and found all the trajectories that are within $\varepsilon_{S}$ distance to it. However, in this setting, we aim at finding similar trajectories by first allowing a pair of translation/rotation to minimize the distance. We used the trajectories from the Cook County data set, and varied the $\varepsilon_{S}$ between 0.5 and 2.5 miles over 200 different choices of $T r_{p i c k}$ and observed the average selectivity. As shown in Figure 7, Steiner-point based routes-matching still introduces a large number of false negatives by up to $60 \%$, since it fails to consider the speed-value, reflected in the temporal-dimension of the trajectories' points.

\section{RELATED WORK}

The problems of matching geometric shapes and pointsets are of interest for various applications areas: computer vision, pattern recognition, time-series-analysis, molecular biology, speech recognition and image processing [5, 17]. A survey of approaches for various geometric transformations and morphings including: (1.) rigid transformations (translations + rotations); (2.) homotheties (translations + scaling); (3.) general affine transformations that can occur in projections is presented in [5]. Initially, most of the efforts were focused on transformations that would minimize the Hausdorff distance between the two sets [3, 21, 17] for both cases of optimal and approximate matching. In particular, [3] introduced the concept of a reference point, which is the Steiner point for compact 2D polygons, and proposed an approximate matching algorithm with a loss factor of $1+4 / \pi$. However (c.f. Section 2), for many applications, the Hausdorff distance is not an appropriate distance-measure, and efforts were geared towards the more dynamics-flexible and much harder to compute, Fréchet distance [6]. Specifically, [6] demonstrates that under Fréchet distance, a reference point of loss-factor 2 exists for matching polygonal curves under translations. The variations of the traditional Fréchet distance investigated in [4] pertain to the specific class of the $\kappa$-straight curves, in which the length of any arc is at most $\kappa$ times their Euclidian distance. In such cases, Hausdorff-based algorithms can be used to approximate the Fréchet one. On the other hand, [7] considers $\kappa$-bounded and backbone curves, and introduces efficient approximation and pseudo-output-sensitive exact algorithms that calculate the discrete-Fréchet distance. A general discussion on various distance-measures for point sets and their computations is presented in [16]. Our work builds upon all these results, but its distinct nature is in the introduction of an intuitive distance-function for moving objects trajectories, which takes into consideration the practical assumption in MOD - the objects are moving with the constant speed along individual segment. As we demonstrated, the $T E_{u d}$ a special case of the general Fréchet distance which enables minimization algorithms for point-sets fitting $[22,17]$ to be applied to efficiently obtain the trajectories' similarity under rigid transformations.

Recent efforts from the MOD and GIS communities have targeted the problems of similarity of spatial and spatiotemporal mobility patterns $[9,20,14,28,29]$. In particular, [14] proposed a Finite State Automaton based algorithm which, given a zone-based partition of the spatial domain, reduces the problems of routes and trajectories matching to the problem of recognizing regular expressions. A somewhat similar basis (discretizing the $2 \mathrm{D}$ space) was used in $[9,20]$ in order to address the processing of various spatiotemporal pattern queries, where the trajectories were represented as strings and the query patterns were detected 
based on string-matchings. Although the works have addressed larger categories of queries than we do, e.g., subsections of trajectories matching whereas we consider at most a containment of a shorter-trip into the longer-trip trajectory, our work uses a more intuitive distance-measure and does not require a discretization of the spatial dimension. More recent extensions [28, 29] proposed similarity measures based on the Longest Common Subsequence (LCSS) and addressed the problem of indexing large datasets for the purpose of the efficient evaluation of the similarity-based spatio-temporal queries. We have not addressed the problem of indexing in this work, however, we proposed a different distance function and we took into consideration the rotation in the spatial dimension. Recent results have connected the DTW with finding short-paths in combinatorial manifolds and relating it to the way light travels in media with different coefficients of refraction [15]. In this context, our approach enables an even further reduction of the number of elements in the dynamic-programming based matrix for calculating the paths in the manifolds. Similarity search for trajectories using data reduction (SVD, DFT, wavelets) is presented in [1], however the assumption is that the trajectories are already rotation-aligned.

\section{CONCLUSIONS AND FUTURE WORK}

We addressed the problem of matching moving objects trajectories, where rigid transformations, i.e., translation in spatial and temporal domain, as well as rotation in the spatial domain, can be applied in order to detect the similarity of their motion. We used an intuitive distance function $T E_{u d}$ and we demonstrated that it is a special case of the Fréchet distance. Taking this into consideration along with the fact that in typical MOD settings the trajectories are assumed to have constant speed along individual trajectorysegments, we proposed both optimal and approximate algorithms for determining the translations and rotations that will minimize the respective $R T S D / T C S D$ similarity distances of two trajectories. There are several immediate extensions of our work. Firstly, we plan to adapt our results to the, so called, decision problem, which is, given two trajectories, and a constant $\varepsilon$ does there exist transformation that will yield a similarity distance $\leq \varepsilon$ ? An important issue is the actual similarity-based query processing for massive trajectories' datasets, which ultimately demands an efficient indexing structures $[28,29]$. Another avenue that we are planning to pursue is exploring the map-available data when the objects are constrained to move on road networks [18] and see whether some pre-processing can be done that will speed up the calculations of the similarity distances among trajectories, especially for real-time tracking data [30].

\section{REFERENCES}

[1] Rakesh Agrawal, Christos Faloutsos, and Arun N. Swami. Efficient similarity search in sequence databases. In FODO, 1993.

[2] A. Alt, C. Knauer, and C. Wenk. Comparison of distance measures for planar curves. Algorithmica, 38, 2004.

[3] H. Alt, O. Aicholzer, and G. Rote. Matching shapes with a reference point. In Symp. Computational Geometry, 1994.

[4] H. Alt, A. Efrat, G. Rote, and C. Wenk. Matching plannar graphs. Journal of Algorithms, 49, 2003.

[5] H. Alt and L. Guibas. Discrete geometric shapes: Matching, interpolation, and approximation. In Handbook of Computational Geometry, 1999.
[6] H. Alt, C. Knauer, and C. Wenk. Matching polygonal curves with respect to the fréchet distance. In $S T A C S$, 2001.

[7] B. Aronov, S. Har-Peled, C. Knauer, Y. Wang, and C. Wenk. Fréchet distance for curves, revisited. In ESA, 2006.

[8] F. Aurenhammer. Voronoi diagrams - a survey of a fundamental geometric data structure. ACM Comput. Surv., 23(3), 1991.

[9] P. Bakalov, M. Hadjielefteriou, and V. Tsotras. Time relaxed spatiotemporal trajectory joins. In GIS, 2005.

[10] Thomas Brinkhoff. A framework for generating network-based moving objects. GeoInformatica, 6(2), 2002

[11] H. Cao, O. Wolfson, and G. Trajcevski. Spatio-temporal data reduction with deterministic error bounds. VLDB Journal, 15(3), 2006.

[12] M. de Berg, M. van Kreveld, M. Overmars, and O. Schwarzkopf. Computational geometry: algorithms and applications. Springer-Verlag New York, Inc., 1997.

[13] S. E. Dreyfus. An appraisal of some shortest - path algorithms. Operations Research, 17(3), 1969.

[14] C. du Mouza and R. Rigaux. Multi-scale classification of moving objects trajectories. In SSDBM, 2004.

[15] A. Efrat, Q. Fan, and S. Venkatasubramanian. Curve matching, time warping, and light fields: New algorithms for computing similarity between curves. J. Mathematic Imaging and Vision, 2007. to appear.

[16] T. Eiter and H. Mannila. Distance measures for point sets and their computation. Acta Inf., 34(2), 1997.

[17] M.T. Goodrich, J.S.B. Mitchell, and M.W. Orletsky. Approximate geometric pattern matching under rigid motions. IEEE Trans. Pattern Anal. Mach. Intell., 21(4), 1999.

[18] R.H. Güting, V.T. de Almeida, and Z. Ding. Modeling and querying moving objects in networks. $V L D B J ., 15(2)$, 2006.

[19] R.H. Güting and M. Schneider. Moving Objects Databases. Morgan Kaufmann, 2005.

[20] M. Hadjielefteriou, G. Kollios, P. Bakalov, and V. Tsotras. Complex spatio-temporal pattern queries. In $V L D B, 2005$.

[21] P.J. Heffernan and S. Schirra. Approximate decision algorithms for point set congruence. In Symp. Computational Geometry, 1992.

[22] K. Imai, S. Sumino, and H. Imai. Minimax geometric fitting of two corresponding sets of points and dynamic furthest voronoi diagrams. IEICE Trans. Inf. and Syst., E81-D(11), 1998.

[23] Forbes Online Issue http://www.forbes.com/home, April 2006.

[24] F. P. Preparata and M. I. Shamos. Computational Geometry: an introduction. Springer Verlag, 1985.

[25] J. Schiller and A. Voisard. Location-based Services. Morgan Kaufmann Publishers, 2004.

[26] M. Sharir and P. K. Agarwal. Davenport-Schinzel Sequences and Their Geometric Applications. Cambridge University Press, 1995.

[27] G. Trajcevski, O. Wolfson, K. Hinrichs, and S. Chamberlain. Managing uncertainty in moving objects databases. ACM TODS, 29(3), 2004.

[28] M. Vlachos, M. Hadjielefteriou, D. Gunopulos, and E. Keogh. Indexing multidimensional time-series. $V L D B$ Journal, 15(1), 2006.

[29] M. Vlachos, G. Kollios, and D. Gunopulos. Elastic translation invariant matching of trajectories. Machine Learning, 58, 2005.

[30] C. Wenk, R. Salas, and D. Pfoser. Addressing the need for map-matching speed: Localizing global curve-matching algorithms. In SSDBM, 2006. 